\title{
Early Repair Processes in Marrow Cells Irradiated and Proliferating in Vivo'
}

\author{
J. E. TILL ANd E. A. McCULLOCH \\ Department of Medical Biophysics, University of Toronto, and the \\ Ontario Cancer Institute, Toronto, Ontario
}

\section{INTRODUCTION}

The radiation survival curves for the proliferative capacity of mammalian cells, both in vitro $(1,2)$ and in vivo $(3,4)$, are sigmoidal, suggesting that loss of proliferative capacity involves an accumulation of radiation damage. For mammalian cells in culture, Elkind and co-workers $(5,6)$ have demonstrated that accumulated sublethal damage may be rapidly repaired in surviving cells, by means of experiments in which the number of survivors was shown to increase when the radiation dose was given as two fractions separated by an interval of time. They have termed this phenomenon "recovery" $(5,7)$, and Elkind ( 7 ) has used the results obtained with cell cultures to predict the outcome of dose-fractionation experiments in vivo. Recently, it has been shown that recovery occurs in irradiated tumor cells in vivo (8). There has, however, been no direct experimental evidence confirming the existence of recovery in normal cells in vivo.

The finding (4) that normal mouse bone marrow contains cells capable of forming macroscopically visible colonies in the spleens of heavily irradiated animals provides a quantitative approach to this problem. An important advantage of the spleen-colony technique is that it may be used to study directly the effects of radiation on cells in vivo (9). In this paper, we report the results of dose-fractionation experiments carried out in this system. The results indicate that early repair of sublethal damage occurs in colony-forming cells irradiated in vivo, but suggest that the time scale of the early repair process differs from that observed for cells in culture.

\section{MATERIALS AND METHODS}

\section{Mice}

$\mathrm{C} 57 \mathrm{Bl} / \mathrm{Ha}$ or $\mathrm{C} 3 \mathrm{Hf} / \mathrm{Ha}$ mice of both sexes, bred in the colony of the Ontario Cancer Institute, or the F1 hybrid between these strains, were used for most experi-

${ }^{1}$ This research was supported, in part, by the Defence Research Board of Canada, under Grant DRB 9350-14 ( $\mathrm{G}$ and $\mathrm{C}$ ) and in part by the National Cancer Institute of Canada. 
ments. For some experiments, Swiss mice from the same colony were used. The techniques used in the marrow transplantation procedure were similar to those described previously $(4,9)$. In the course of this work, it was found that anomalous results may be obtained if the mice used as donors of marrow cells and the mice used as heavily irradiated recipients of marrow transplants were not of identical strains. In particular, the use of C57Bl marrow for transplantation into C57Bl $\times$ $\mathrm{C} 3 \mathrm{H}$ F1 hybrids yielded unusually low colony counts, as will be discussed in a subsequent publication.

\section{Irradiation Techniques}

The irradiation procedures were similar to those previously described (9). Irradiation of recipient mice was carried out with 280-kvp X-rays, HVL $1.12 \mathrm{~mm} \mathrm{Cu}$, at an exposure dose rate of approximately $150 \mathrm{r} / \mathrm{min}$. For some experiments, 200kvp X-rays, HVL $1 \mathrm{~mm} \mathrm{Cu}$, were used. For irradiation of marrow cells, $\mathrm{Co}^{60} \gamma$-rays were used at a dose rate of about $50 \mathrm{rads} / \mathrm{min}$. Dosimetry was based on air ionization measurements made with a Philips vibrating-reed electrometer and on absorbed dose measurements made with the ferrous ammonium sulfate dosimeter (10).

\section{Irradiation of Colony-Forming Cells in Vivo}

The method for measuring the number of cells in a marrow suspension with the capacity to give rise to colonies in the spleen has been described (4). The modification of the technique to allow the irradiation of these colony-forming cells after their implantation within the animal has also been described (9). In brief, in the in vivo method, the recipient animal receives a portion of the large dose of wholebody radiation required to suppress the formation of colonies from endogenous cells (see below). After this, the cells are injected, and a period of time in excess of 1 hour is allowed, as this interval has been found to be sufficient to allow the cells to manifest the radiation sensitivity characteristic of the in vivo situation (9). After this interval, a second dose of whole-body radiation is given with $\mathrm{Co}^{60}$ $\gamma$-rays. The two doses are so arranged that their sum is 950 rads. However, while the recipient animal receives the total dose of radiation, the injected cells receive only the dose delivered in the second exposure. Evidence has been presented to show that the injected cells respond to irradiation in vivo by this technique in a quantitatively similar way to cells irradiated in situ (9). In order to study the effect of dose fractionation, this design needs only to be modified by delivering the dose, after the injection of the cells, in two parts, with an interval of time between them.

An example will illustrate the method used in dose-fractionation experiments. If the cells of a transplant are to receive two doses of 200 rads with an interval between the doses, the following procedure is used: First the animals receive 550 rads of X-rays. Then, an appropriate number of marrow cells are injected intravenously in $1 / 2 \mathrm{ml}$ of suspending fluid. After an interval of 1 hour, the animals receive 400 rads of $\mathrm{Co}^{60} \gamma$-rays in two equal doses separated by the desired interval of time. 
Ten days after the second $\gamma$-ray dose, the animals are killed, their spleens are fixed in Bouins' solution, and the number of macroscopic colonies visible in the fixed spleen is determined.

\section{Endogenous Colony Formation}

In the example described above, the cells of the transplant received a total of 400 rads of $\gamma$-rays, and the recipient animals received a total dose of 950 rads of $\mathrm{X}$ - and $\gamma$-rays. The latter high dose is required to suppress the appearance of colonies in the spleen due to survival of the animals' own colony-forming cells, and it is sufficiently high to yield an average of less than one "endogenous" colony per spleen even under the conditions of dose fractionation used in the experiments reported in this paper. Lower doses allow the appearance of such endogenous colonies. The number of endogenous colonies visible after 11 days is related to radiation dose in the manner to be discussed under Results (see Fig. 4). Cobalt-60 $\gamma$-rays were used in all studies of the effect of radiation on endogenous colonies.

\section{RESULTS}

\section{Cell Survival after Fractionated Doses in Vivo}

The dose-response curve for colony-forming cells after a single dose of $\gamma$-rays in vivo has been published (9). This curve. shown in Fig. 1, is characterized by a $D_{0}$ of $95 \pm 9$ rads and an extrapolation number of 1.5. The effect of dose fractionation is shown in Fig. 2. Curve $b$ shows the results obtained when the interval between the doses is 3 to 4 hours. Curve $a$ is the single-dose curve shown in Fig. 1. For the fractionation experiments, a constant first dose of 200 rads was chosen, as the survival after this dose falls on the straight-line portion of the survival curve. Therefore, the survival curves obtained by dose fractionation are shown originating from this point. It is apparent from curve $b$ that considerable restoration of the shoulder region occurred during the 3 - to 4 -hour interval. The results of curve $c$ will be described later.

\section{The Kinetics of the in Vivo Repair Process}

Figure 3 shows the results obtained when two fixed doses of 200 rads each were given to colony-forming cells in vivo, separated by different intervals of time. The ordinate of Fig. 3 is the survival ratio, defined as the ratio of the percentage of surviving colony-forming cells after two doses separated by a time interval, $t$, to the percentage survival for the same total dose given as a single exposure. The abscissa gives the time interval, $t$, between the two doses.

The smooth curve drawn through the points of Fig. 3 shows an initial maximum at about 5 hours, followed by an intermediate minimum at about 11 hours. The survival ratio at the 5 -hour maximum appears to be greater than would be expected 


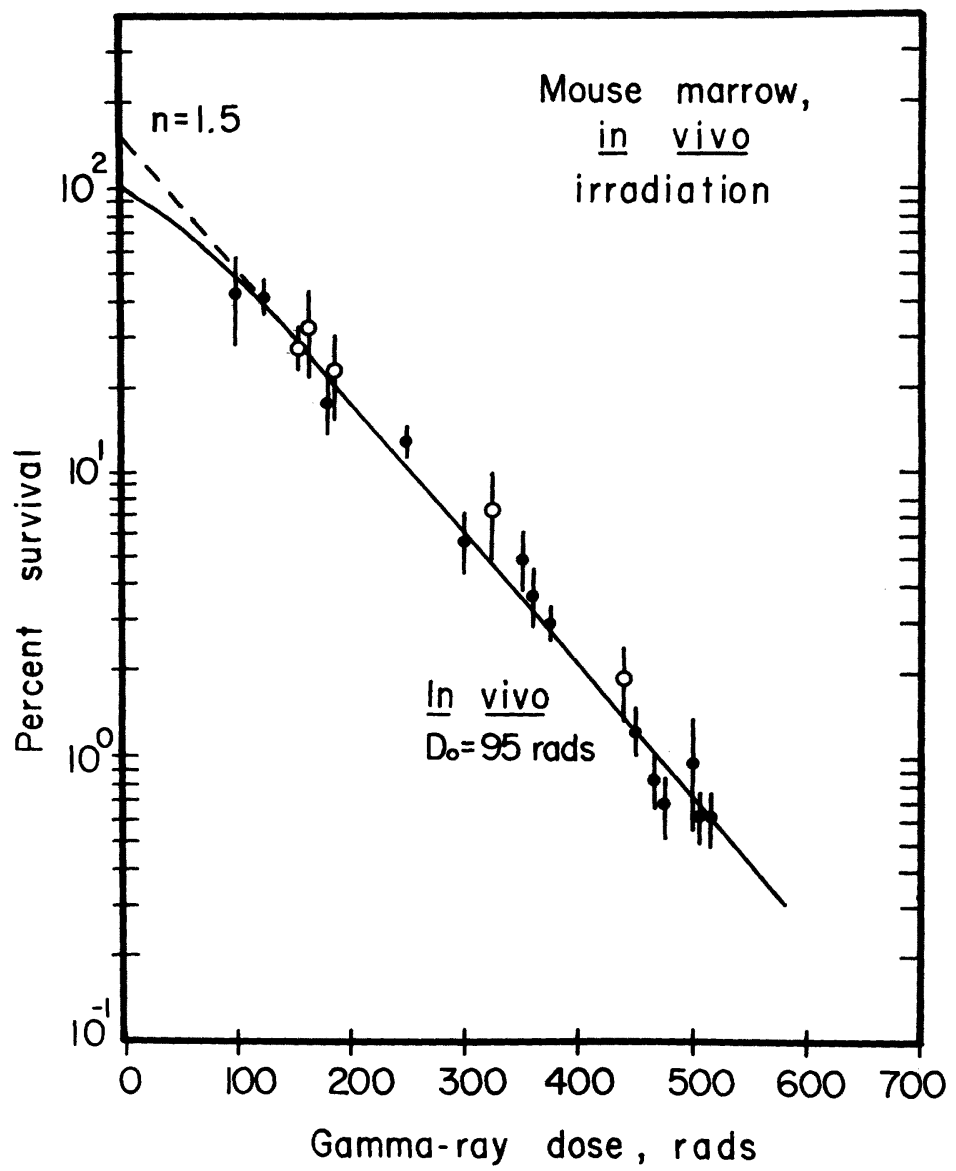

FIg. 1. Survival curve for colony-forming cells in mouse bone marrow transplants irradiated in vivo with a single dose of $\mathrm{Co}^{80} \gamma$-rays (9).

from the extrapolation number of 1.5 for the single-exposure survival curve (Fig. 1). However the difference is not statistically significant $(P>0.2)$.

\section{Validity of the Intermediate Minimum}

The intermediate minimum at about 11 hours could result from a change in the slope of the survival curve rather than a change in extrapolation number. In order to test for this possibility, a survival curve was constructed for an 11-hour interval between the doses. A first dose of 200 rads was again used. The results of two experiments, shown in Fig. 2, curve $c$, indicate that the intermediate minimum is in fact due to a failure of the shoulder region of the survival curve to reappear after dose fractionation with an 11-hour interval. 


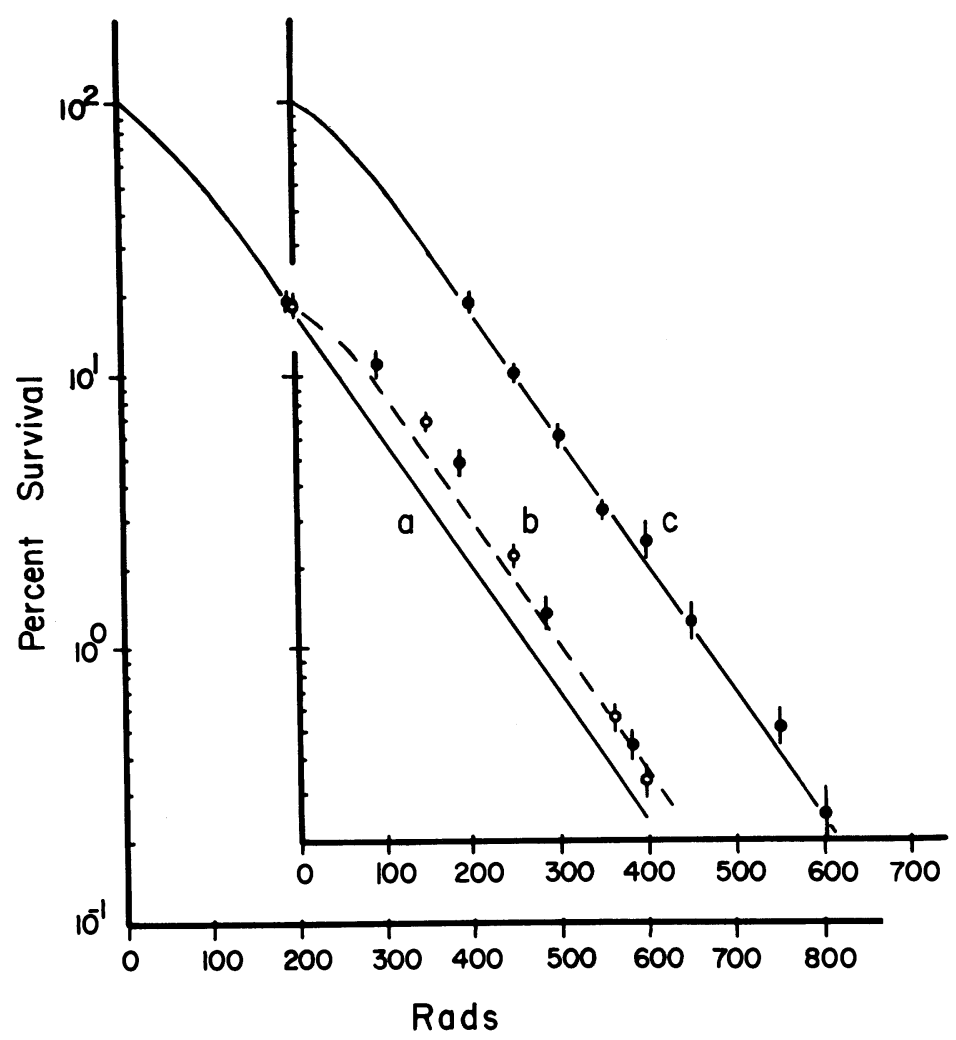

Fig. 2. Survival curve for colony-forming cells from mouse marrow irradiated in vivo with two doses of $\mathrm{Co}^{60} \gamma$-rays separated by an interval of time. Curve $a$ (lower axes): in vivo survival curve, see Fig. 1. Curve $b$ (lower axes) : closed circles, 3-hour interval between doses; open circles, 4-hour interval between doses. Curve $c$ (upper axes): 11-hour interval between doses. Results of two separate experiments. Standard errors for the individual points are shown.

\section{Repair in Endogenous Colony-Forming Cells}

The results described above show that early repair processes occur in transplanted marrow cells irradiated in vivo. Additional experiments were carried out to show that similar processes occur in cells irradiated in situ. These experiments were based on the observation that colonies are seen in the spleens of mice 11 days after irradiation with 600 to 800 rads, in the absence of a marrow transplant. The number of endogenous colonies observed is related to radiation dose in the manner illustrated in Fig. 4, in which the mean number of colonies per spleen of total-bodyirradiated C57Bl mice is plotted against the indicated $\mathrm{Co}^{60} \gamma$-ray dose. Exponential curves each having a $D_{0}$ of 95 rads have been drawn through the experimental points. It is evident that the radiation sensitivity of colony-forming cells in situ is 


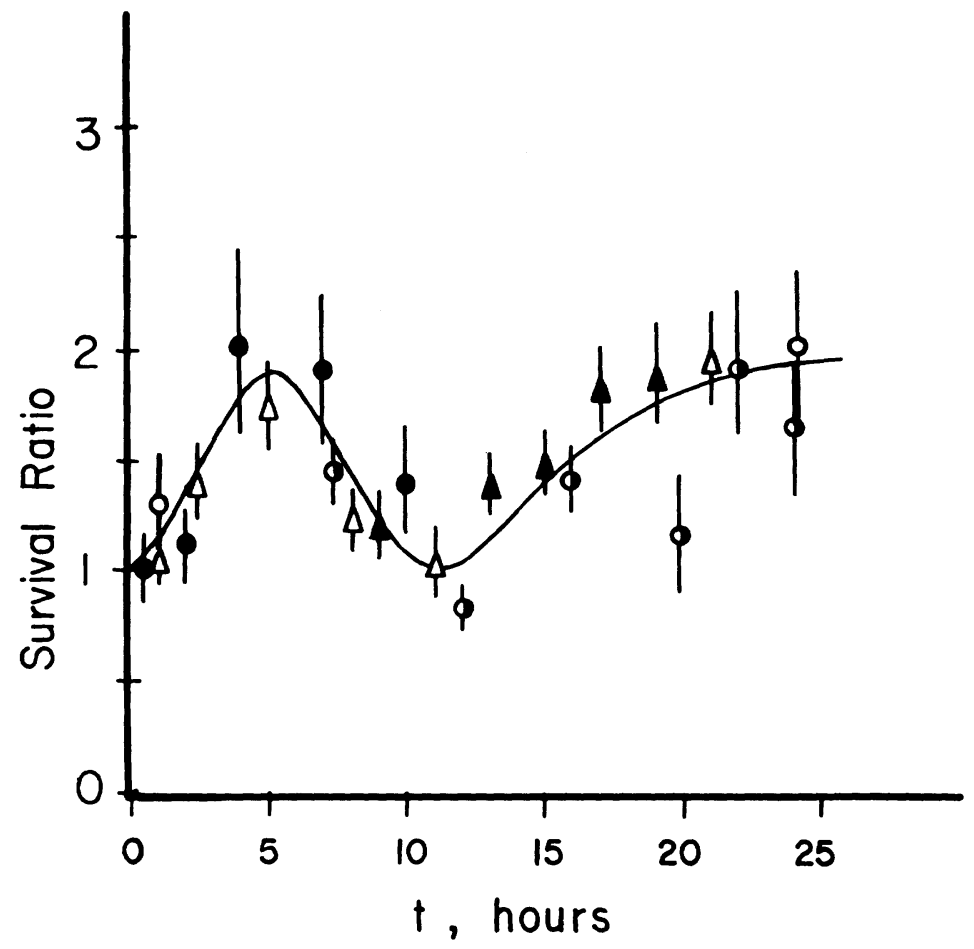

FIg. 3. Kinetics of early repair of colony-forming cells from mouse marrow irradiated in vivo with two 200-rad doses of $\mathrm{Co}^{\infty 0} \gamma$-rays separated by different time intervals $(t)$. Ordinate: survival ratio (see text). Abscissa: time interval between the doses. The different symbols indicate separate experiments. Standard errors for the individual points are shown.

similar to the radiation sensitivity of transplanted colony-forming cells (Fig. 1). It is also apparent that, in this particular experiment, a higher level of surviving endogenous colony-forming cells was found in females than in males. This difference, which preliminary experiments indicate may be due to a difference in the number of colony-forming cells in the spleens of males and females, has no detectable influence on the results of experiments in which the transplantation technique is used, since, in isologous hosts, the sex of neither donor nor recipient has been found to influence the survival of colony-forming cells.

Applying this method to a test of the effect of dose fractionation on endogenous colony-forming cells yielded the results shown in Fig. 5. The figure shows the time dependence of the survival ratio of endogenous colony-forming cells for the particular pair of doses used (first dose, 400 rads; second dose, 300 to 350 rads). Though the statistical spread of the data is quite severe, the existence of an initial maximum and an intermediate minimum in the kinetic curve, similar to that shown in Fig. 3, is apparent. The survival ratio observed in these experiments was 


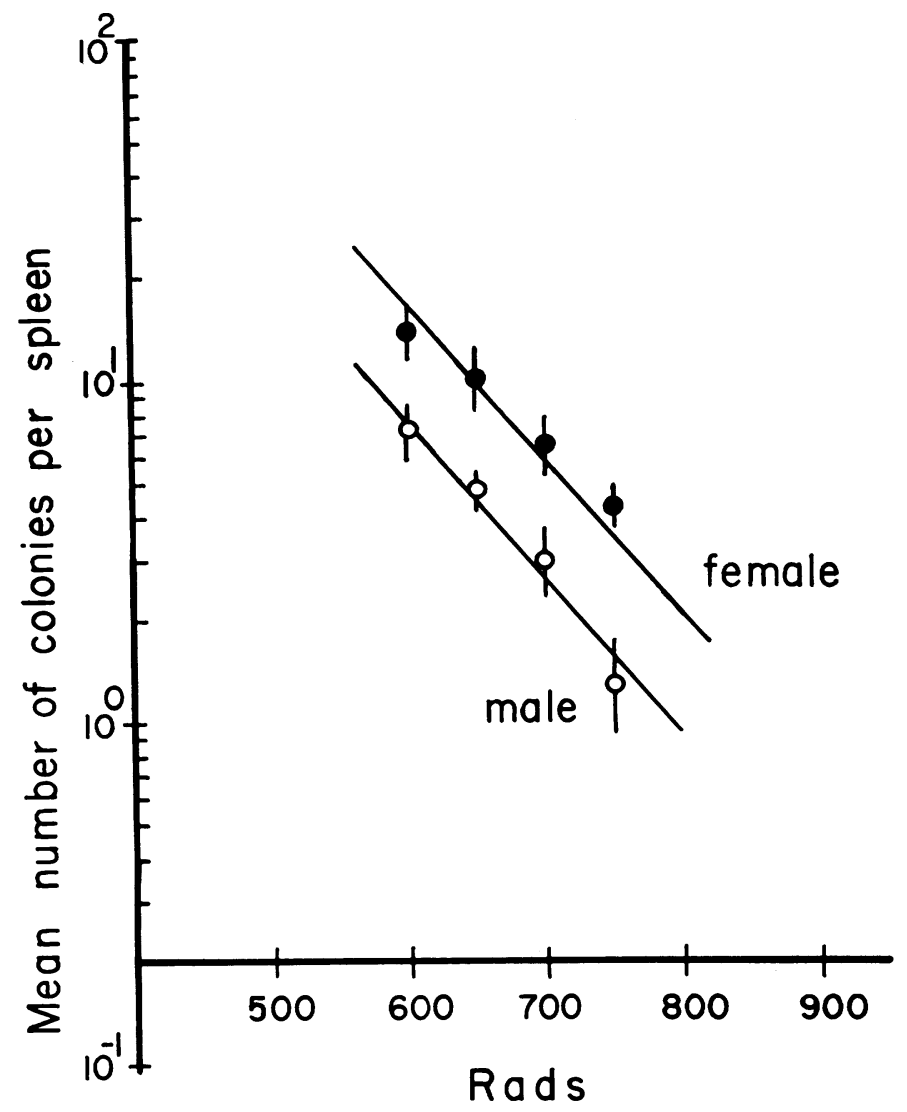

Fig. 4. Survival curve for endogenous spleen colony-forming cells of C57Bl/Ha mice irradiated in situ with total-body $\mathrm{Co}^{60}$ radiation. Closed circles: female mice. Open circles: male mice. Standard errors are shown.

consistently much greater than that obtained with marrow transplants. The reasons for this difference are unknown, but it is possible that they result from additional repair processes not active in marrow transplants.

\section{DISCUSSION}

The results presented above indicate that a phenomenon analogous to that studied by Elkind and co-workers in Chinese hamster (5) and HeLa (6) cells in culture also occurs in vivo in colony-forming cells of the mouse hematopoietic system.

Comparison of the in vivo results with those of Elkind and co-workers shows qualitative agreement in the forms of the kinetic curves, but the initial maximum and intermediate minimum are shifted in time (about 5 and 11 hours, respectively, 


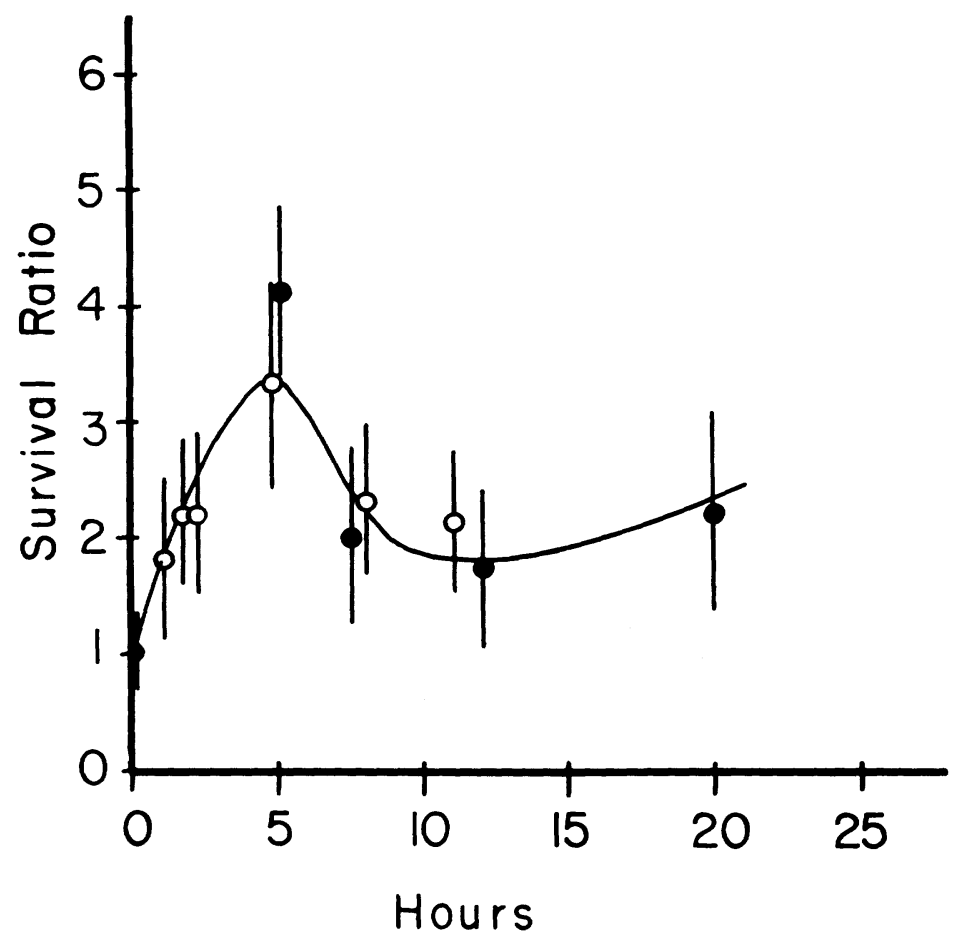

Fra. 5. Kinetics of early repair of endogenous colony-forming cells irradiated in situ with two doses of total-body $\mathrm{Co}^{60}$ radiation. First dose, 400 rads. Closed circles: second dose, 300 rads; open circles: second dose, $\mathbf{3 5 0}$ rads. Standard errors for the individual points are shown.

from cells from mouse marrow, compared with about 2 and 5 hours, respectively, for Chinese hamster cells, 5). In Ehrlich ascites tumor cells in vivo, Hornsey and Silini (8) found recovery to be at its maximum after 5 to 7 hours, with no significant decrease by $91 / 2$ hours, which was the longest time interval used.

In the absence of information concerning the duration of radiation-induced division delay in spleen colony-forming cells in vivo, it is not possible to establish with certainty that the early repair process described above for colony-forming cells is recovery in the sense defined by Elkind ( 7$)$, who has applied the term only to changes in survival occurring during the period of division delay. It is still possible that the changes reported here are the result, in part, of cellular proliferation. This type of change Elkind has termed "repair." We prefer the designation "regeneration" for the effects of cellular proliferation, and in this paper we have used "early repair" as a more general term indicating processes that increase the number of surviving cells during the first day after irradiation. Experiments designed to elucidate the kinetics of proliferation of colony-forming cells in vivo are at present under way. 
Colonies developing in the spleens of mice irradiated with doses between 600 and 800 rads have been used to study the behavior of colony-forming cells in situ. Although experiments designed to study endogenous colony-forming cells yield data of less precision than experiments based on cell transplantation, they have the advantage that they show the behavior of cells without any changes that might be introduced by the transplantation procedures. The experiments reported in this paper indicate that early repair processes occur after whole-body irradiation of the intact mouse. The results also indicate a difference in the number of colonyforming cells in males and females, showing that the method has general application to the study of the physiology of colony-forming cells.

\section{SUMMARY}

1. Early repair of sublethal radiation damage has been demonstrated in surviving spleen-colonizing cells in vivo, by means of dose-fractionation experiments.

2. A study of the kinetics of the early repair process indicated that early repair reached an initial maximum at about 5 hours after the first dose fraction, followed by an intermediate minimum at about 11 hours.

3. An assay procedure which may be used for the study of the behavior of spleencolonizing cells in situ has been developed.

\section{ACKNOWLEDGMENTS}

We wish to thank Miss Rosemary Wyncoll, Mr. James Hicks, and Mr. Paul Csordas for their excellent technical assistance.

ReCEived: June 1, 1962

\section{REFERENCES}

1. T. T. Puck and P. I. Marcus, Action of X-rays on mammalian cells. J. Exptl. Med. 103, 653-666 (1956).

2. T. T. Puck, In vitro studies on the radiation biology of mammalian cells. Progr. Biophys. and Biophys. Chem. 10, 237-258 (1960).

3. H. B. Hewitt and C. W. Wilson, Survival curves for tumor cells irradiated in vivo. Ann. N. Y. Acad. Sci. 95, 818-827 (1961).

4. J. E. Till and E. A. McCulloch, A direct measurement of the radiation sensitivity of normal mouse bone marrow cells. Radiation Research 14, 213-222 (1961).

5. M. M. Elkind and H. Sutton, Radiation response of mammalian cells grown in culture. I. Repair of X-ray damage in surviving Chinese hamster cells. Radiation Research 13, 556593 (1960).

6. R. Z. Lockart, Jr., M. M. Elkind, and W. B. Moses, Radiation response of mammalian cells grown in culture. II. Survival and recovery characteristics of several subcultures of HeLa S3 cells after X-irradiation. J. Natl. Cancer Inst. 27, 1393-1404 (1961).

7. M. M. Elkind, Radiation responses of mammalian cells. Brookhaven Symposia in Biol. No. 14, 220-245 (1961). 
8. S. Hornsey and G. Silini, Recovery of tumor cells cultured in vivo after X-ray and neutron irradiations. Radiation Research 16, 712-722 (1962).

9. E. A. McCulloch and J. E. Till, The sensitivity of cells from normal mouse bone marrow to gamma-radiation in vitro and in vivo. Radiation Research 16, 822-832 (1962).

10. Report of the International Commission in Radiological Units and Measurements (ICRU) 1959. Natl. Bur. of Standards (US.) Handbook No. 78 (1961). 